\title{
Preparation of Ruthenium Chloride-grafted Zeolite from a Clay Mineral Ore and Studying Their Catalytic Properties
}

\author{
Ragheed Yousif Ghazal ${ }^{* 1}$, Dhyaa Mahmood Fathy *2 \\ $1^{*, 2}$ Department of Chemistry, College of Education for pure Science, University of Mosul, Mosul, Iraq \\ E-mail: ${ }^{1 *}$ ragheedghazal76@uomosul.edu.iq, ${ }^{2}$ Dhyaa.esp96@ student.uomosul.edu.iq
}

(Received July 26, 2021; Accepted September 14, 2021; Available online December 01, 2021)

DOI: 10.33899/edusj.2021.130894.1178, (c) 2021, College of Education for Pure Science, University of Mosul. This is an open access article under the CC BY 4.0 license (http://creativecommons.org/licenses/by/4.0/)

\begin{abstract}
This research work include studying one of the natural mineral ores available in Al-Hawy area (Mosul city - Northern Iraq) by chemical analysis and X-ray fluorescence (XRF) to identify its components of the elements as oxides, X-ray diffraction was carried out to determine the percentages of clay minerals (natural zeolites) and non-clay minerals in the natural ore. The natural zeolites were concentrated by removing carbonate, iron and separating the convertible silica into sodium silicate, the prepared zeolite was converted into ( $\mathrm{H}$-form) by treated with ammonium nitrite solution $(1 \mathrm{M})$, then grafted with ruthenium chloride $\left(\mathrm{RuCl}_{3} .6 \mathrm{H}_{2} \mathrm{O}\right)$. The properties and specifications of the prepared zeolite (grafted and non-grafted) were studied using techniques of (XRF) ,(XRD),(BET), (SEM) and (differential\& thermo gravimetric analysis(DTA)\&(TGA) ), it was found that have a chemical and crystalline composition within the specifications of zeolites, as well as a good surface area, thermal stability and selective porous channels.
\end{abstract}

Keywords: zeolite, montmorillonite, kaolinite, SEM, XRD

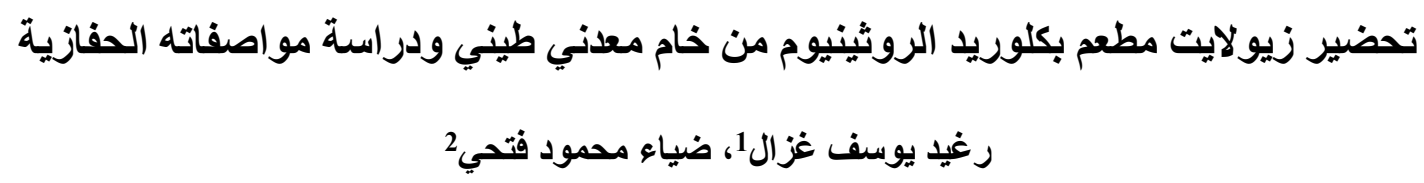

1*2قسم الكيمياء، كلية التربية للعلوم الصرفة، جامعة الموصل، الموصل، العراق

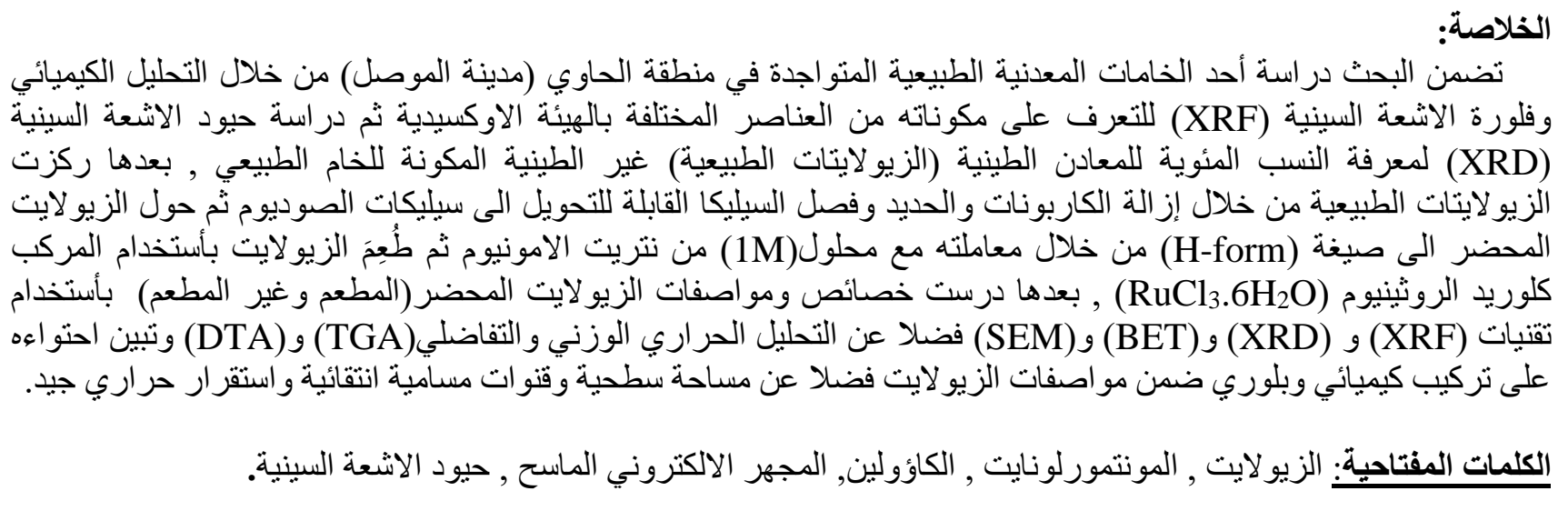




\section{Journal of Education and Science (ISSN 1812-125X), Vol: 30, No: 5, 2021 (103-113)}

المقدمة

يعرف الزيولايت كيميائياً بانه عبارة عن سيليكات الألمنيوم المائية المتبلورة ويعد العالم السويدي (Cronstadt) أول من

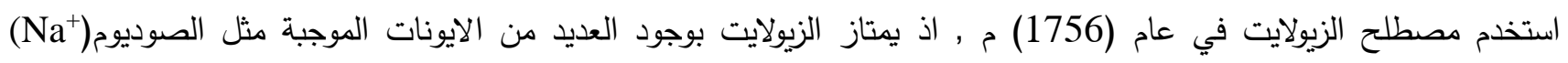

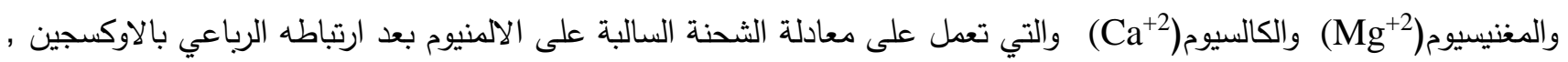
وان طريقة الارتباط بين عنصري الالمنيوم والسيليكون هي التي تحدد التركيب البلوري المميز لكل نوع من انواع الزيولايت المختلفة(1), حيث تتشا الزيولايتات من اتحاد الوحدات الرباعية الاوجه للسيليكون مع الوحدات الرباعية الإنية الاوجه للالمنيوم (الوحدات

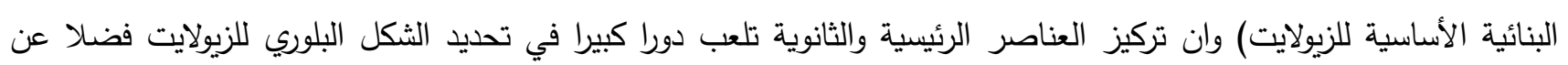
حجم المسامات (التجاويف الداخلية المرتبطة بالسطح الخارجي) في كل نوع من انواع الزيولايتات , اما الوحدة البنائية الثانوية

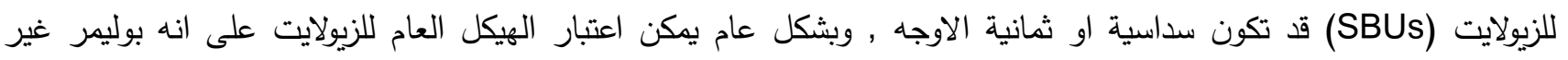

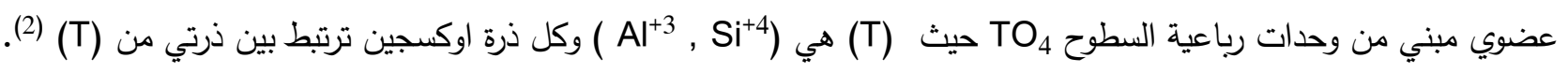

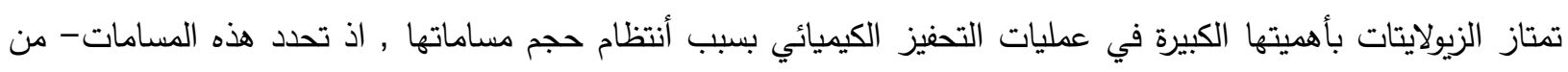

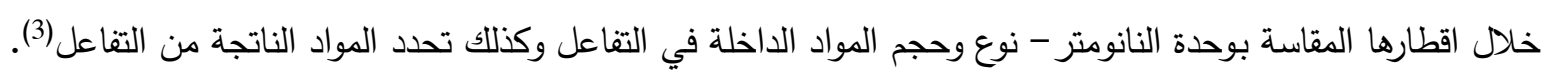
على الرغم من امتلاك الزيولايتات مواصفات عديدة ومهمة الا ان الباحثين لجأوا الى تحويره كيميائيا وذلك لجعله اكثر فعالية ,

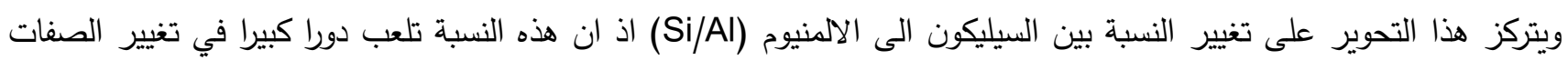

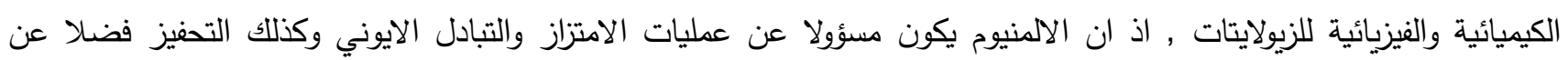
الثباتية الكيميائية و الحرارية للزيولايت , لذا فانه من المهم جدا تعديل هذه النسبة مع المحافظة على التركيب البلوري للهيكل الخاص بالزيولايت (4).

وقد اهتم الباحثين بشكل كبير بدراسة الزيولايتات بانواعها المختلفة بسبب الخصائص الكيميائية والفيزيائية واهمها حامضية برونشتد وحامضية لويس والاستقرار الحراري و قدرتها على الامتزاز والمساحة السطحية الكبيرة نسبياً وقدرتها على التبادل الايوني, , وهناك ميزات اخرى للزيولايتات اذ تمتلك كثافة واطئة وقدرة عالية على التميؤ فضلا فلا عن المكانية اعادة تتشيطها بسهولة وباقل كلفة) و (6,5).

وهناك العديد من الدراسات المتضمنة تحضير الزيولايتات وتطبيقاتها في مجال الصناعات البتروكيمياوية: اذ قام (Yu) وجماعته سنة (2020) بتحضير زيولايت نوع (Y) المطعم بالزركونيوم واطلق عليه رمز (ZrUSY) بعملية

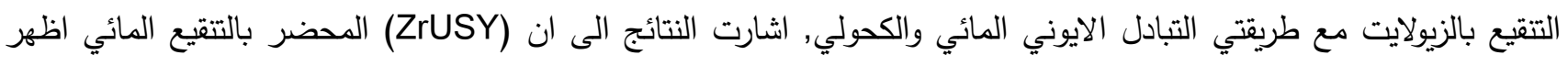

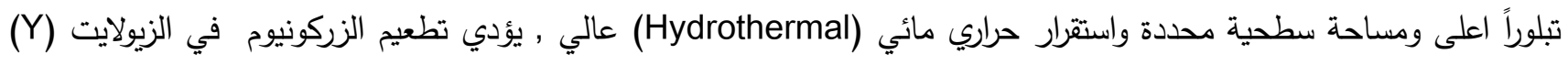

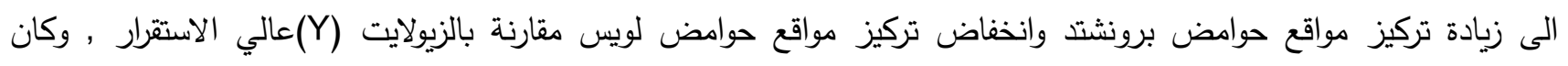
(ZrUSY) انتقائية لتكوين الاوليفينات , ودرس (Delgado)(8) واخرون سنة (2020) امكانية تحضير الزيولايت من نفايات الالمنيوم الخطرة باستخدام مفاعل الاوتوكليف ذو سعة (200) لتر في ظل ظروف تشغيل حرارية معتدلة في عملية من خطوة واحدة اذ يمكن انتاج

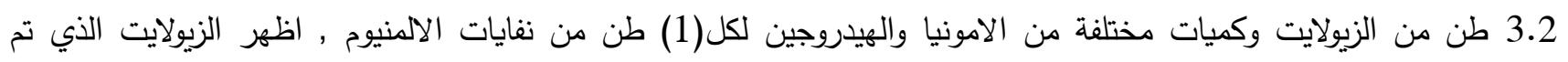

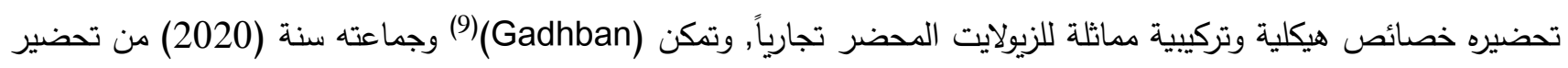

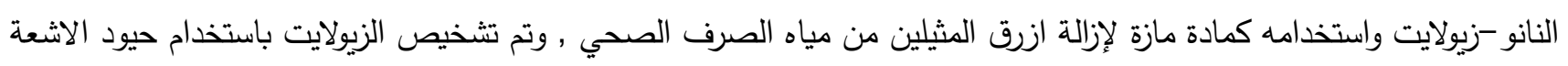
السينية (XRD) ولوحظ ان الزيولايت النانوي كان نشطا في ازالة ازرق المثلين الذي يعد من الملوثات الثائعة لمياه الصرف الصناه الصحي في المنشآت الصناعية, و استطاع (Lee)(10) وجماعته سنة (2020) تصنيع محفزات الزيولايت(ZSM-5 (ZSM) باستخدام نسب مختلفة من العامل الموجه للبنية والسيليكا والالومينا , واستخدمت نماذج (ZSM-5) في تفاعل التحلل الماص للحرارة (2) 


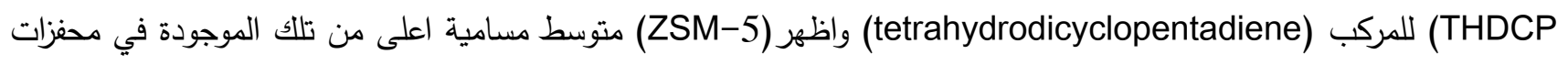

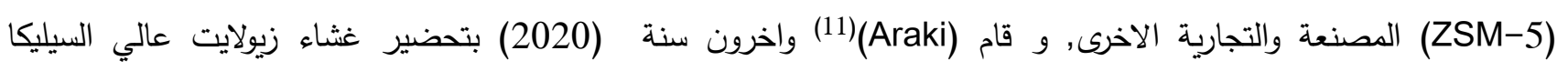

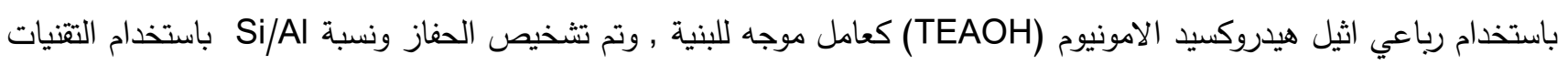

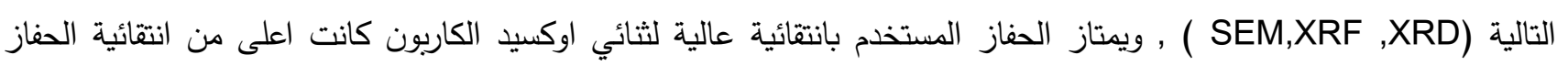
المحضر بالطرق الاخرى.

\section{الجزء العملي \\ 1 - اختيار ودراسة الخام المعدني الطيني قيد البحث:}

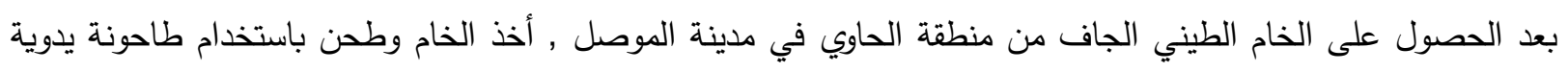

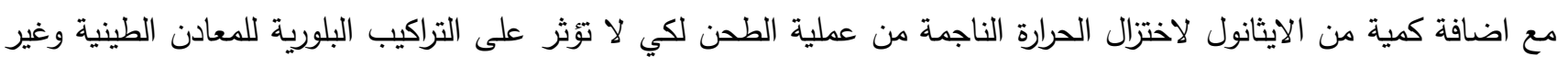

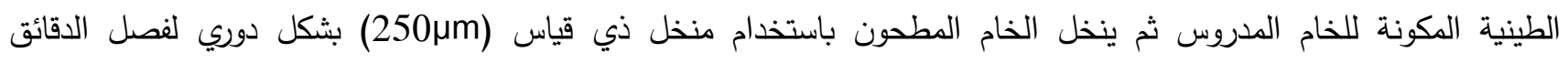

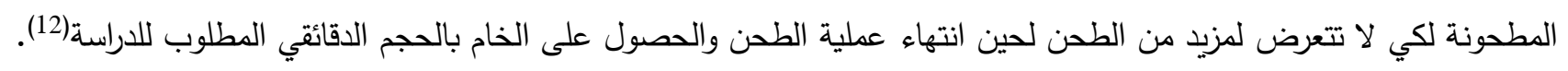

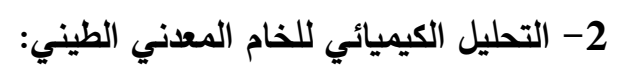
أجري التحليل الكيميائي للخام الطيني الجاف قيدين الدئين الدراسة بعد صهره باستخدام زيج متكافئ من هيدروكسيد وكاربونات

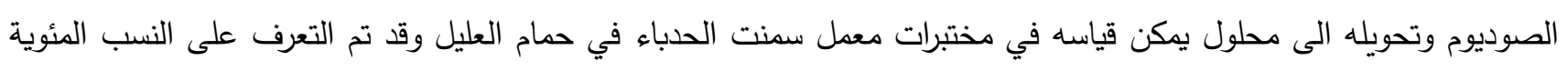

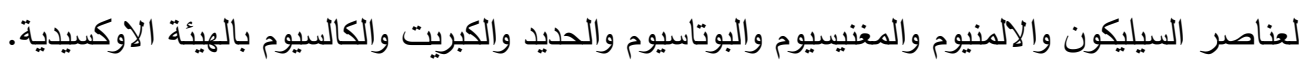

3- تحضير الزيولايت

1 إزالة الكاربونات من الخام الطيني (150)

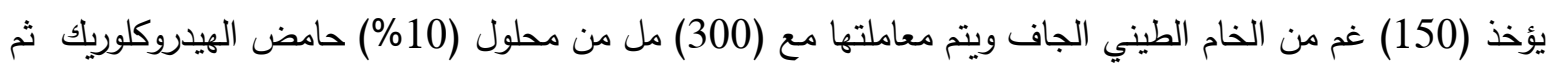

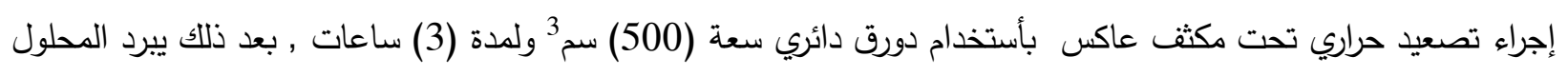

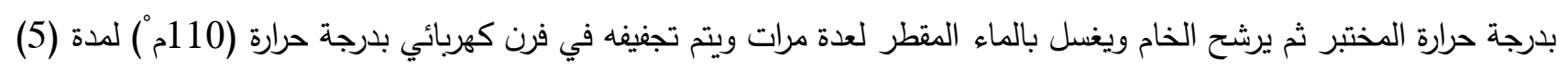

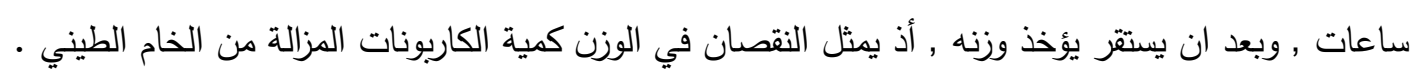

3 2 إزالة الحديد من الخام الطيني(15)

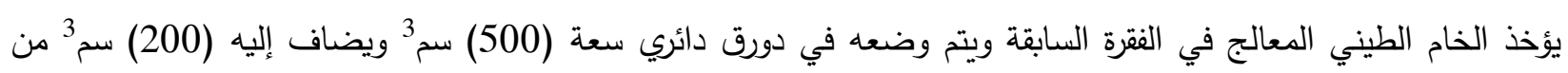

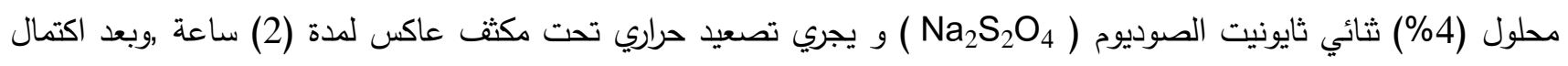

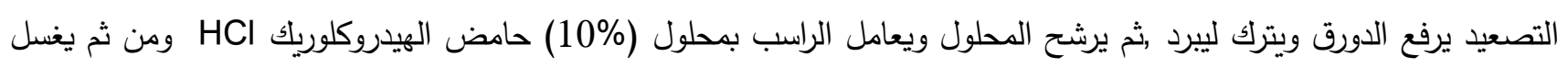

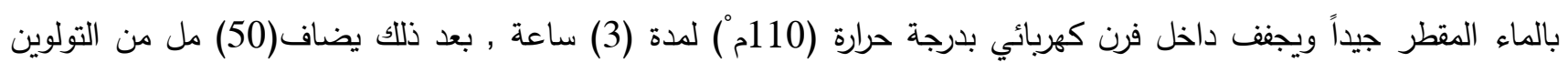

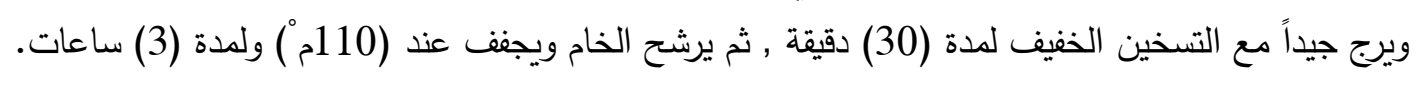

(16) 3.3 فصل السيليكا من الخام الطيني يؤخذ نموذج الخام المعالج في النقرتين السابقتين, حيث يوضع نموذج الخام الطام الطيني في دورق دائري سعة (500)سم3 ويضاف

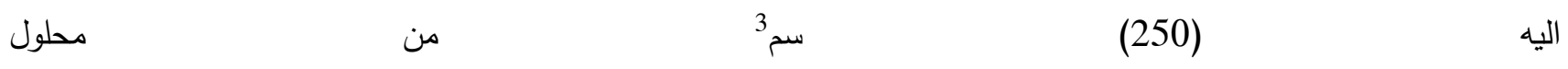
(0.5) مولاري هيدروكسيد البوتاسيوم , ويصعد حراريا تحت مكثق عاكس لددة (5) ساعات ويترك الدورق لييرد بدرجة حرارة

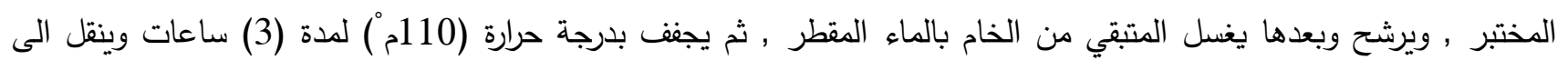


المجفف (Desiccator) وبعد ان يستقر يؤخذ الوزن , ويمثل النقصان في الوزن كمية السيليكا القابلة للتحويل الى سيليكات الصوديوم • بعد هذه الخطوة تكتمل تتقية الزيولايت الموجود في الخام المعدني الطيني وسيكتمل تحضيره بشكل نهائي في الخطوتين اللاحقتين.

4.3 تحويل الزيولايت المحضر الى صيغة (17) H- Zeolite). يؤخذ الزيولايت المحضر في الفقرة السابقة ويضاف اليه (150) مل من محلول (1) مولاري نتريت الامونيوم (25) حيث يوضع في دورق دائري سعة (250) سم3 ويصعد حراريا باستخدام مكثف عاكس ولمدة (2) ساعة, بعدها يبرد المحلول بدرجة ملإية حرارة المختبر ويرشح وبعدها يغسل بالماء المقطر عدة مرات , ثم يؤخذ الراسب (الزيولايت ) ويجف في فرات فرن كهربائي عند درجة

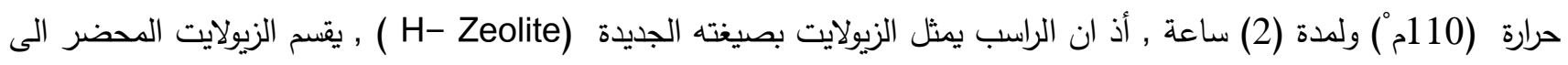
قسمين ليتم تطعيم نصفه في الخطوة اللاحقة.

5.3 تطعيم الزيولايت المحضر بكلوريد الروثينيوم(18)

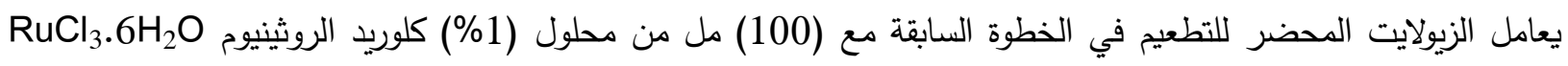
حيث يوضع في دورق دائري سعة (250) سم3 ويصعد حراريا تحت مكثف عاكس ولمدة (3) ساعات , ثم يبرد بدرجة حرارة

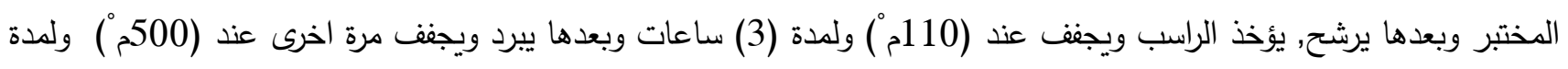
• (3) ساعات اخرى

\section{3}

لغرض التعرف على مواصفات الزيولايتات المحضرة المطعم وغير المطعم أجري عدد من القياسات وهي فلورة الاشعة السينية وحيود الاشعة السينية (XRF) و (XRD) و القياس بتقنية (BET) والقياس بالمجهر الالكتروني الماسح (SEM) فضرفيلا عن التحليل

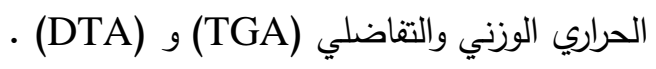

\section{النتائج والمناقشة}

1- تحليل الخام الطيني قيد الاراسة

بعد اجراء التحليل الكيميائي للخام الطيني تم الحصول على النتائج الددرجة في الجدول (1) الجدول (1) التحليل الكيميائي للعناصر الموجودة في الذام الطيني بالصيغة الاوكسيدية

\begin{tabular}{|c|c|}
\hline Metal Oxides & Percentage Ratio (\%) \\
\hline $\mathrm{SiO}_{2}$ & 50.9 \\
\hline $\mathrm{Al}_{2} \mathrm{O}_{3}$ & 17.9 \\
\hline $\mathrm{MgO}$ & 7.1 \\
\hline $\mathrm{K}_{2} \mathrm{O}$ & 2.7 \\
\hline $\mathrm{Fe}_{2} \mathrm{O}_{3}$ & 5.23 \\
\hline $\mathrm{SO}_{3}$ & 0.5 \\
\hline $\mathrm{CaO}$ & 12.41 \\
\hline L.O.I & 3.2 \\
\hline
\end{tabular}


يتبين من الجدول اعلاه احتواء الخام الطيني قيد الدراسة على نسبة عالية من السيليكا (50.9\%) وهي من المواد الاساسية المكونة للزيولايت مع الالومينا التي كانت نسبتها (17.9\%) فضلا عن ذلك احتواء الخام الطيني على تراكيز مختلفة من الكالسيوم والحديد والمغنيسيوم , وكانت نسبة الفقدان بالحرق( L.O.I) (3.2\%).

2- قياس خصائص ومواصفات الزيولايت المحضر بعد تحضير الزيولايتين الغير المطعم والمطعم بكلوريد الروثينيوم تم اجراء العديد من القياسات عليهما للتعرف على لئل الخصاص والمواصفات لكل نوع من خلال قياس فلورة الاشعة السينية (XRF) و حيود الاشعة السينية (XRD) وتئنية الامتزاز بطريقة (BET) ومجهر المسح الاككتروني (XEM) فضلا عن اجراء التحليل الحراري الوزني والتفاضلي. ومن خلال قياس فلورة الاشعة السينية(XRF) وكما مبين في الجدول (2) تبين ان الزيولايت المحضر (الغير المطعم بكلوريد الروثينيوم) يتكون بصورة اساسية من عنصري السيليكون والالمنيوم ونسب مختلفة من بقية العناصر وان نسبة السيليكون الى فئين الالمنيوم تقترب من (1.42) وهو يتوافق مع النسب المئوية للسيليكون والالمنيوم في الخام لكل من المعدنين الطينيين( الكاؤولين والمونتمورلونايت).

جدول (2) النسب المئوية لأكاسيد العناصر المتواجدة في الزيولايت الطبيعي (غير المطع بكلوريد الروثينيوم) من خلال فلورة الأثعة السينية (XRF) .

\begin{tabular}{|c|c|}
\hline Metal Oxides & Wt.(\%) \\
\hline $\mathrm{SiO}_{2}$ & 62.59 \\
\hline $\mathrm{Al}_{2} \mathrm{O}_{3}$ & 21.993 \\
\hline $\mathrm{MgO}$ & 6.75 \\
\hline $\mathrm{K}_{2} \mathrm{O}$ & $\mathbf{3 . 0 3}$ \\
\hline $\mathrm{Fe}_{2} \mathrm{O}_{3}$ & 0.9487 \\
\hline $\mathrm{TiO}_{2}$ & 0.92 \\
\hline $\mathrm{CaO}$ & $\mathbf{0 . 1 5 1}$ \\
\hline L.O.I & 3.31 \\
\hline
\end{tabular}

اما نتائج حيود الاشعة السينية (XRD) للزيولايت الطبيعي (الغير مطعم بكلوريد الروثينيوم) فكانت كما في الشكل (1): carts

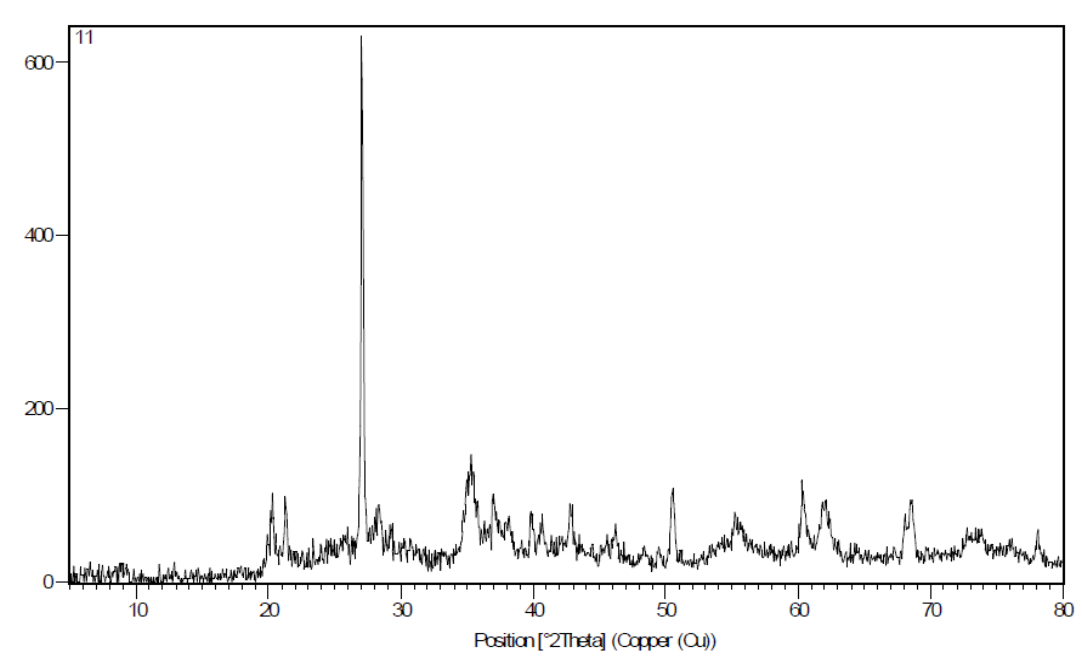

الثكل (1) حيود الاشعة السينية (XRD) للزيولايت الطبيعي (الغير مطعم بكلوريد الروثينيوم) 
يتبين من الثكل(1) ان الزيولايت المحضر من الخام الطبيعي ومن خلال قيم زوايا الحيود (theta) لكل نمط من انماط الحيود ومقارنتها مع اشكال الحيود القياسية (19) لكل معدن من المعادن الطينية وغير الطينية ان الزيولايت يتكون اساساً من المعادن المبينة في الجدول(3) معنون

الجدول (3) النسب المئوية للمعادن المتواجدة في الزيولايت المحضر من خلال قياس (XRD)

\begin{tabular}{|c|c|c|}
\hline Zeolite constituents & Chemical structures & Wt.(\%) \\
\hline Montmorillonite & $(\mathrm{Na}, \mathrm{Ca})_{0.33}(\mathrm{Al}, \mathrm{Mg})_{2}\left(\mathrm{Si}_{4} \mathrm{O}_{10}\right)(\mathrm{OH})_{2} \cdot \mathrm{nH}_{2} \mathrm{O}$ & 39.42 \\
\hline Quartz & $\mathrm{SiO}_{2}$ & 36.76 \\
\hline Kaolinite & $\mathrm{Al}_{2}\left(\mathrm{Si}_{2} \mathrm{O}_{5}\right)(\mathrm{OH})_{4}$ & 23.01 \\
\hline Calcite & $\mathrm{CaCO}_{3}$ & 0.54 \\
\hline Hematite & $\mathrm{Fe}_{2} \mathrm{O}_{4}$ & 0.27 \\
\hline
\end{tabular}

يتبين من خــلال الجــدول (3) ان هذا الزيولايت المحضر من الخام المعدني الطيني يحتوي على ما نسبته (62.43\%) من

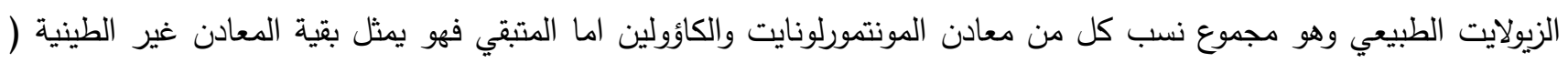
الكوارتز والكالسايت والدولومايت).

ومن خلال قياس فلورة الاشعة السينية(XRF) تبين ان الزيولايت المحضر (المطعم بكلوريد الروثينيوم) يتكون بصورة اساسية

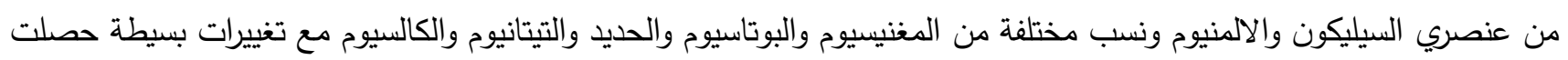
بعد التطعيم كما موضح في الجدول (4). الجدول (4) النسب المئوية لأكاسيد العناصر المتواجدة في الزيولايت (المطعم بكلوريد الروثينيوم) من خلال فلورة الأثشعة السينية (XRF).

\begin{tabular}{|c|c|}
\hline Metal Oxides & Wt.(\%) \\
\hline $\mathrm{SiO}_{2}$ & 61.90 \\
\hline $\mathrm{Al}_{2} \mathrm{O}_{3}$ & 21.331 \\
\hline $\mathrm{MgO}$ & 6.96 \\
\hline $\mathrm{K}_{2} \mathrm{O}$ & 2.97 \\
\hline $\mathrm{Fe}_{2} \mathrm{O}_{3}$ & 0.8984 \\
\hline $\mathrm{TiO}_{2}$ & 0.84 \\
\hline $\mathrm{CaO}$ & 0.015 \\
\hline L.O.I & 3.2 \\
\hline
\end{tabular}

اما نتائج حيود الاشعة السينية (XRD) للزيولايت الطبيعي ( المطعم بكلوريد الروثينيوم) فكانت كما في الشكل (2): carts

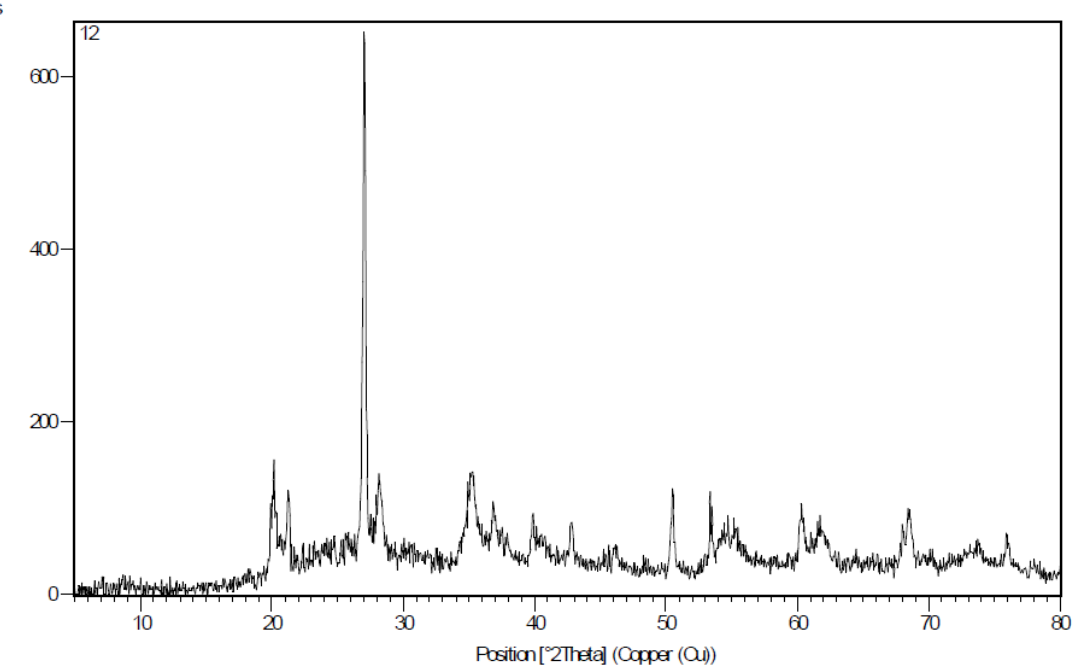

الثكل (2) حيود الاشعة السينية (XRD) للزيولايت الطبيعي (المطع بكلوريد الروثينيوم) 
ومن خلال تقنية (BET) تبين ان الزيولايت المحضر (الغير مطعم بكلوريد الروثينيوم) ذو مواصفات جيدة من حيث

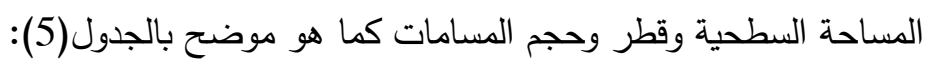

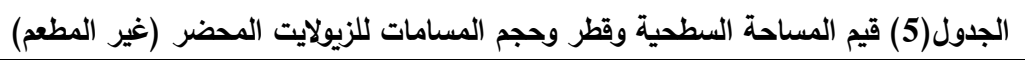

\begin{tabular}{|c|c|}
\hline Measurements & Analysis Data \\
\hline BET Surface Area & $79.0341 \mathrm{~m}^{2} / \mathrm{g}$ \\
\hline Langmuir Surface Area & $556.3289 \mathrm{~m}^{2} / \mathrm{g}$ \\
\hline Pore Volume & $0.1984325 \mathrm{~cm}^{3} / \mathrm{g}$ \\
\hline Pore Size & $6.16710 \mathrm{~nm}$ \\
\hline
\end{tabular}

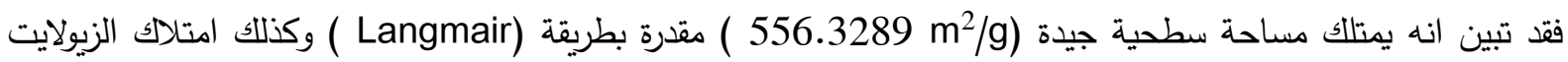

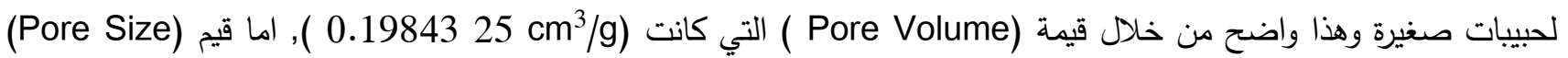

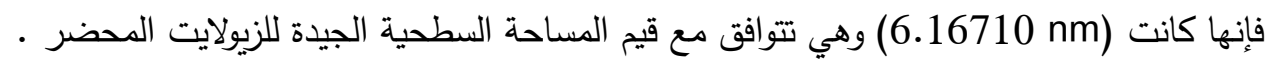

اما فيما يخص الزيولايت المطعم فكانت قيم نتائج قياس (BET) كما موضح بالجدول(6):

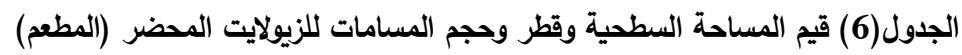

\begin{tabular}{|c|c|}
\hline Measurements & Analysis Data \\
\hline BET Surface Area & $80.8050 \mathrm{~m}^{2} / \mathrm{g}$ \\
\hline Langmuir Surface Area & $518.7893 \mathrm{~m}^{2} / \mathrm{g}$ \\
\hline Pore Volume & $0.1881325 \mathrm{~cm}^{3} / \mathrm{g}$ \\
\hline Pore Size & $5.88593 \mathrm{~nm}$ \\
\hline
\end{tabular}

اذ تبين من خلال نتائج قياس (BET) للزيولايت المحضر (المطعم بكلوريد الروثينيوم) ان هناك اختلافات بسيطة اهمها

نقصان قيمة المساحة السطحية لتصبح 518.7893 m²/g وهذا يمكن تفسيره على ان وجود كلوريد الروثينيوم قد ادى الى صغر حجم المسامات بسبب حجمه الكبير وهذا واضح من خلال نقصان قيمة Pore Size الى (5.88593 ) . واظهرت نتائج قياس المجهر الالكتروني الماسح للزيولايت الغير مطعم بكلوريد الروثينيوم انه يتكون من حبيبات ذات

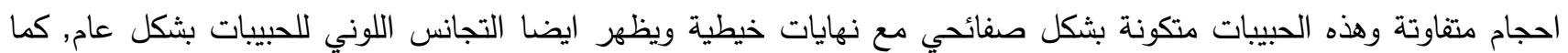
موضح في الثكل (3).

اما فيما يخص نتائج قياس المجهر الاككتروني الماسح للزيولايت المطعم بكلوريد الروثينيوم فقد تبين ان هناك تغييرا

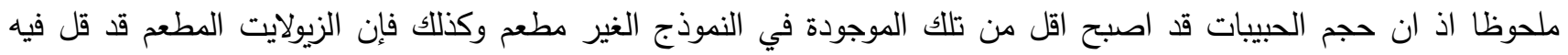
وبشكل واضح النهايات الخيطية ويلاحظ فيه عدم التجانس اللوني من خلال المناطق البيضاء اللون , كما موضح في الثكل (4) . 


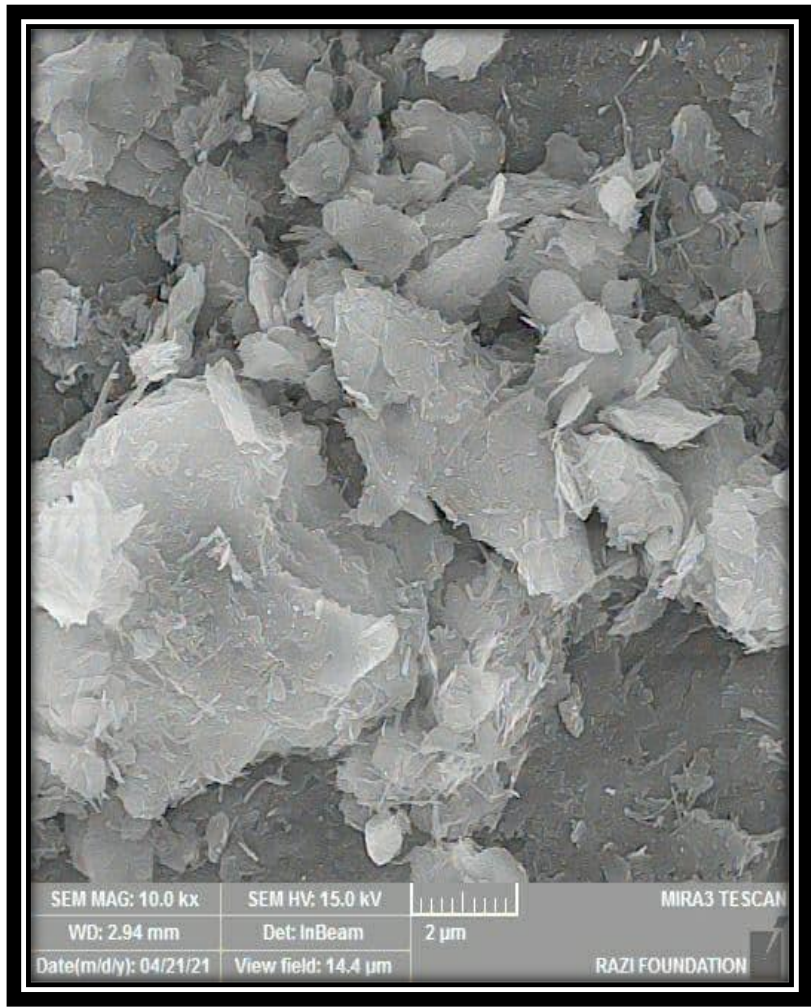

A

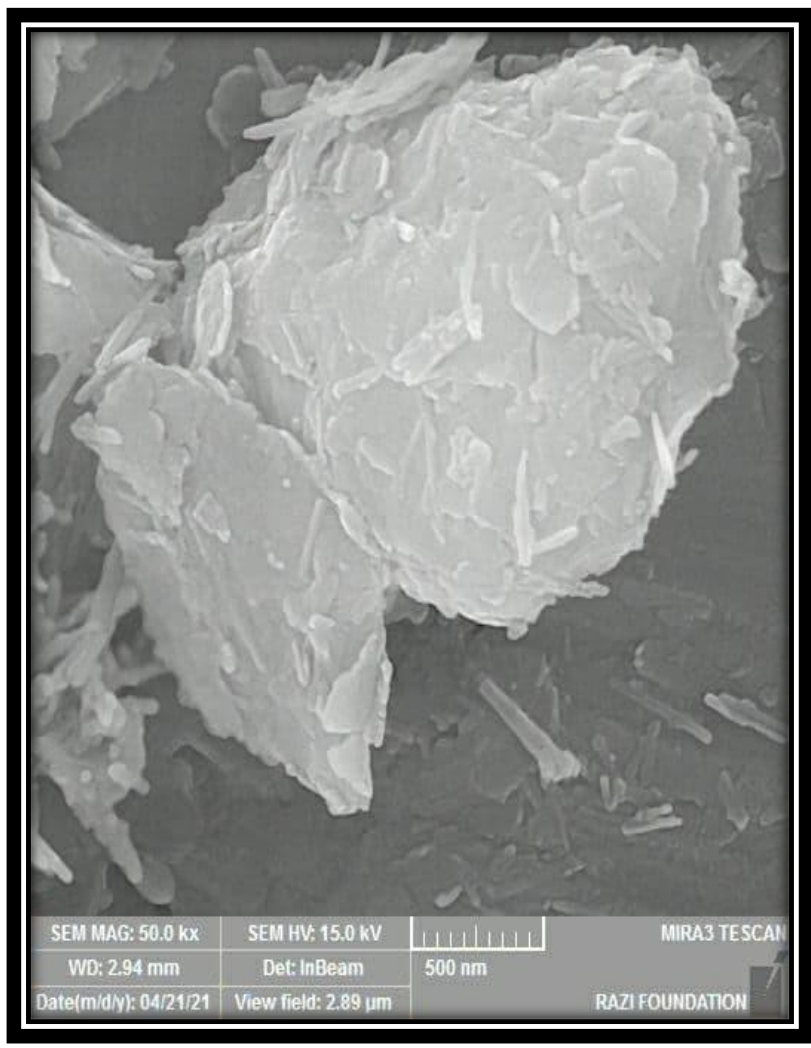

C

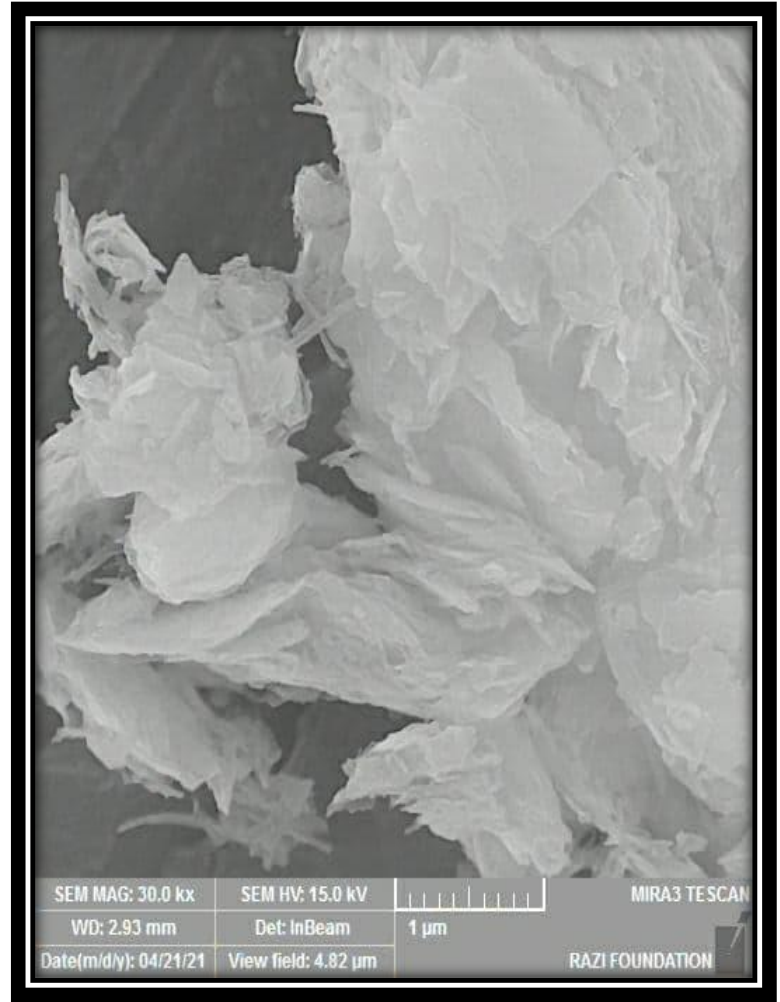

B

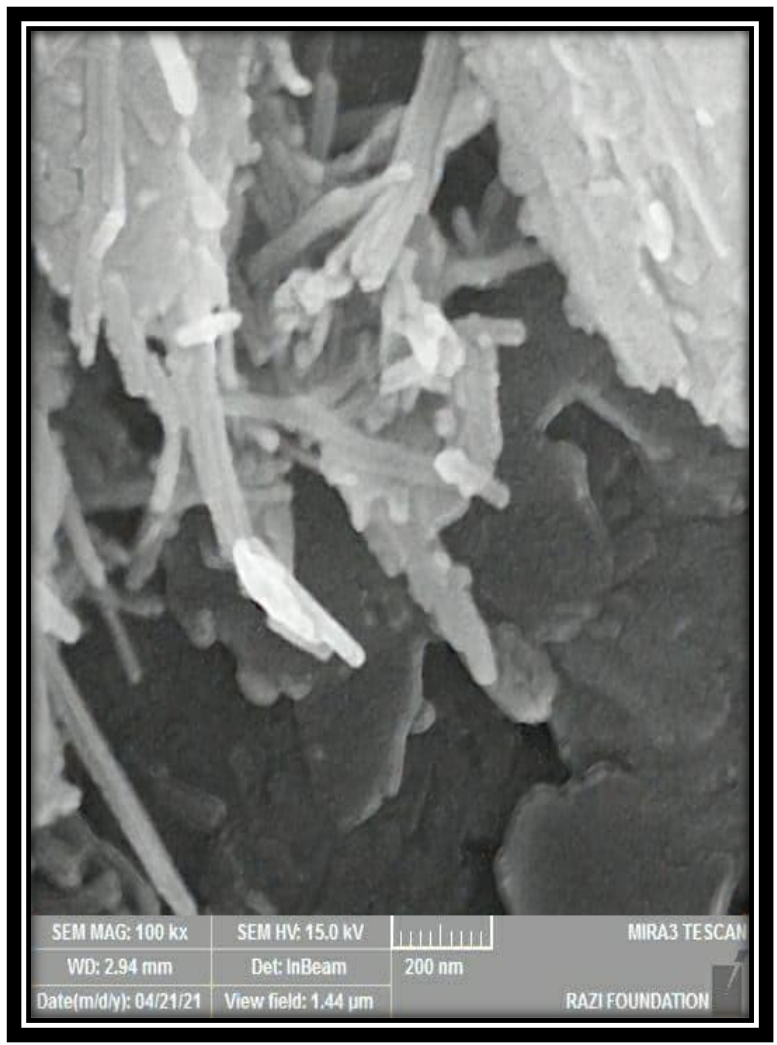

D

B , $2 \mu m$ =A) الثكل (3) صور المجهر الاكتروني بتكبيرات مختلفة للزيولايت المحضر (الغير المطعم بكلوريد الروثينيوم ) ( $200 \mathrm{~nm}=\mathrm{D}, 500 \mathrm{~nm}=\mathrm{C}, 1 \mu \mathrm{m}=$ 


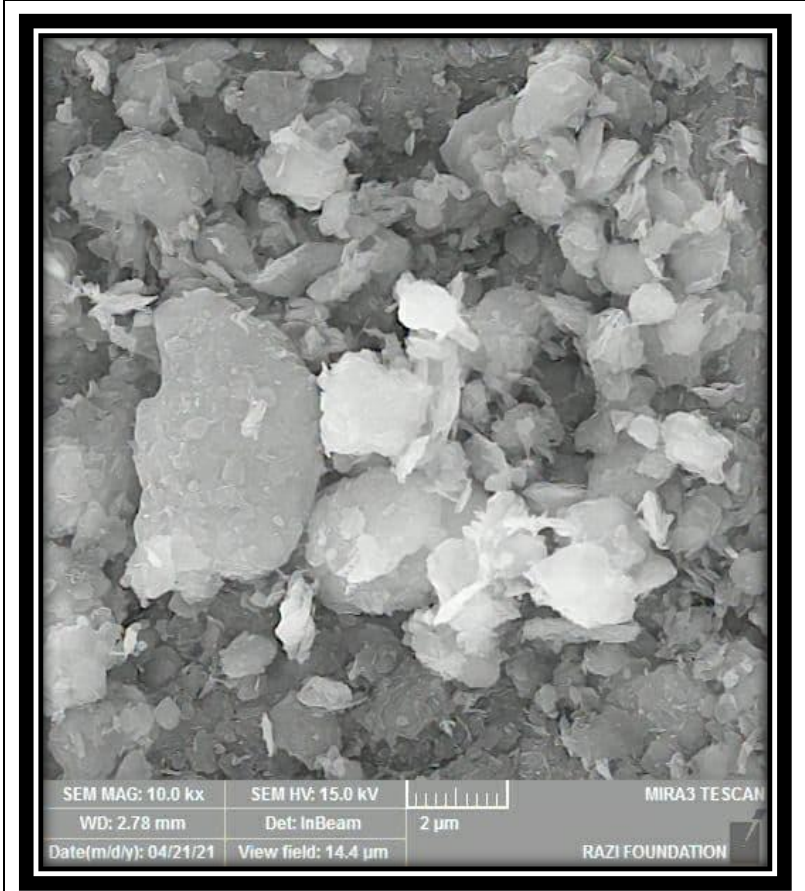

A

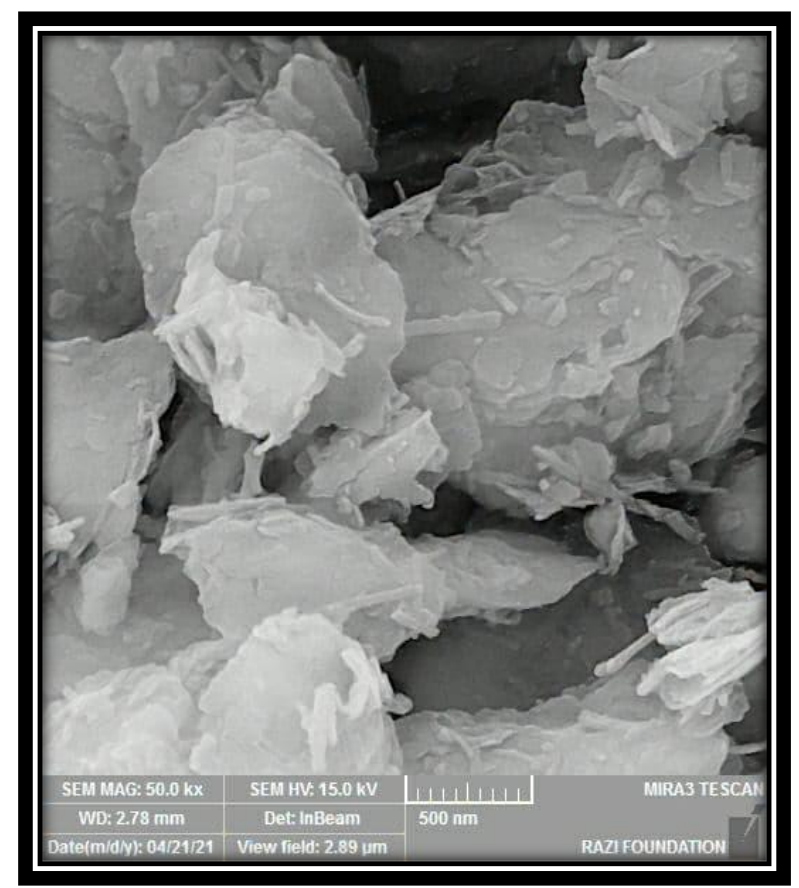

C

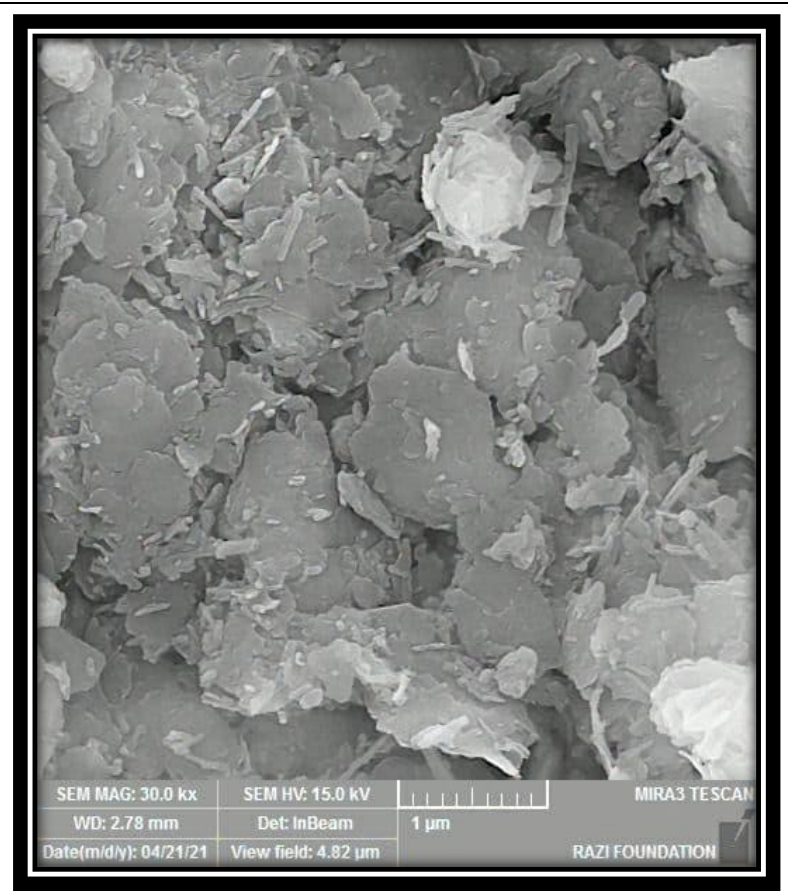

B

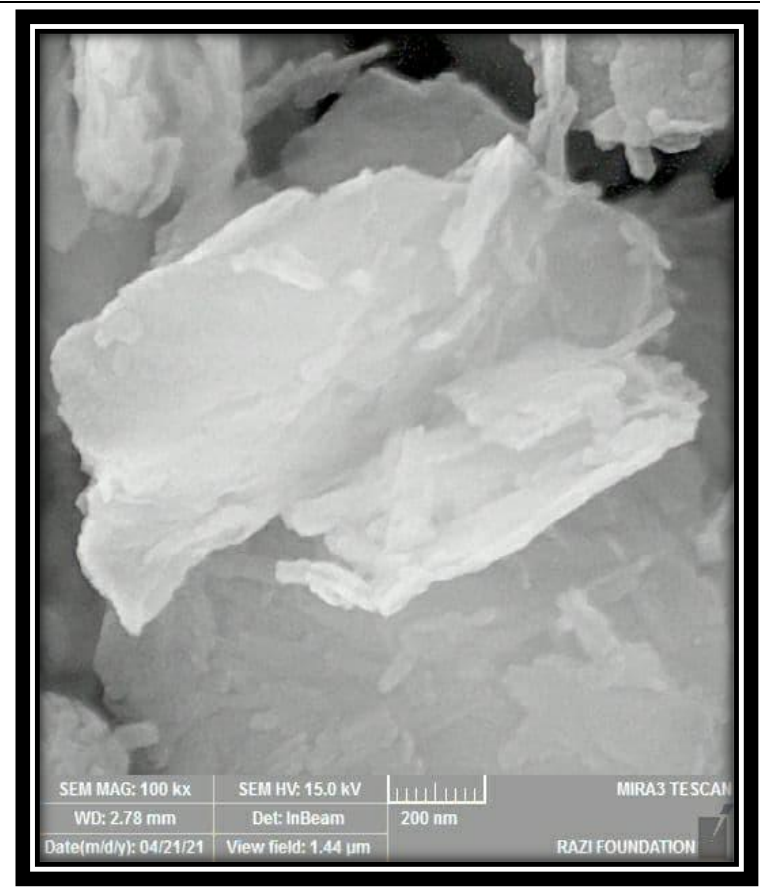

$\mathrm{D}$

=B , الثكل (4) صور المجهر الاكتروني بتكبيرات مختلفة للزيولايت المحضر (المطعم بكلوريد الروثينيوم ) ( $200 \mathrm{~nm}=\mathrm{D}, 500 \mathrm{~nm}=\mathrm{C}, 1 \mu \mathrm{m}$

وعند اجراء التحليل الحراري الوزني والتفاضلي للزيولايت غير المطعم بكلوريد الروثينيوم للتعرف على كمية الماء الموجود في

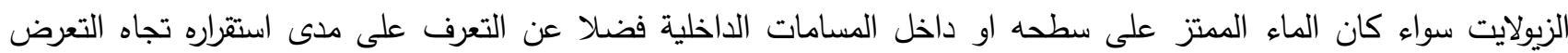
لدرجات الحرارة العالية , اذ بين القياس ان الزيولايت يحتوي على ماء ممتز على سطحه بكمية (3.7) \% عتريبا والتي بدأ فقدانها 
بحدود درجة الحرارة (50) مْ وصولا الى درجة حرارة (150) مْ في حين كانت كمية الماء الموجودة داخل القنوات المسامية تقدر

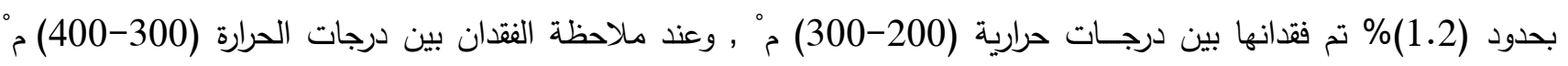

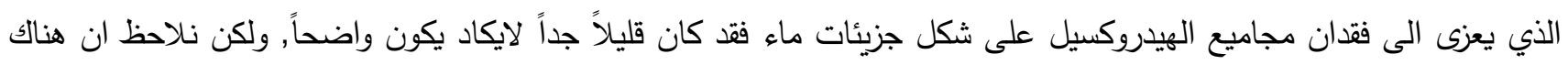

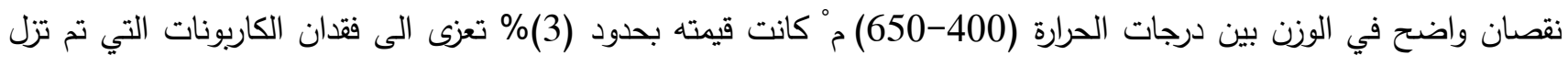
بالكامل وهذا واضح من قيم نسبة المغنسيوم والكالسيوم في الجدول ( 2 و 4) (20) , ويبدو واضحا من خلال التحليل الحراري التفاضلي انه يكون مستقراً لحد درجة حرارة (800) مْ وهذا الكلام ينطبق على الزيولايت المطعم ايضاً لكن بكميات فقدان في الاوزان

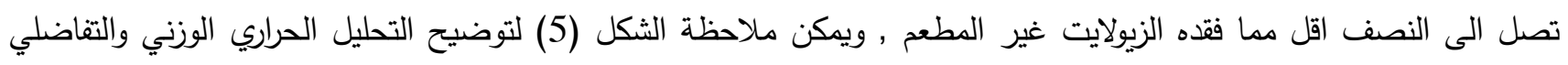

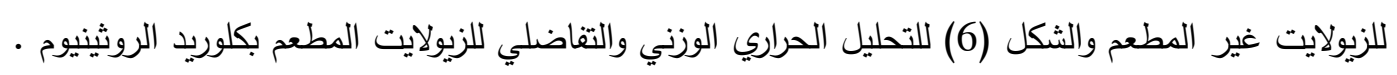
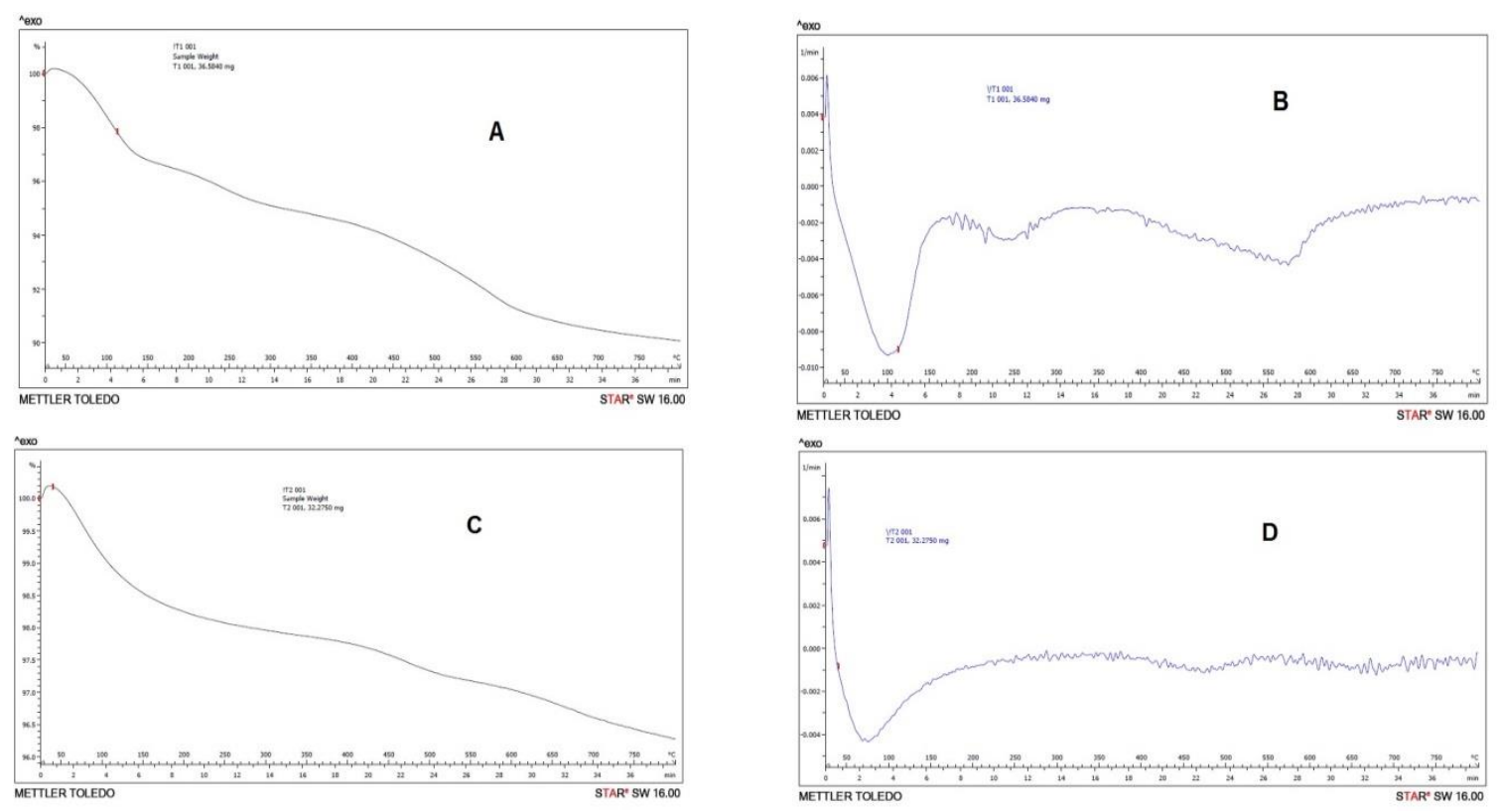

\section{الثكل (5) : (A) A B)(التحليل الحراري الوزني والتفاضلي للزيولايت غير المطعم

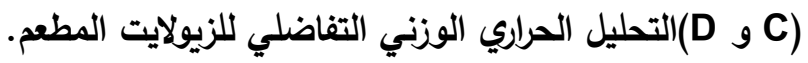

الاستنتاجات

تبين من خلال النتائج ان الزيولايتين المحضرين (الغير مطعم والمطعم بكلوريد الروثينيوم) لهما مساحة سطحية وقنوات مسامية جيدة واستقرار حراري جيد فضلا عن امتلاكهما درجة تبلور وقدرة امتزازية عاليتين يمكن من خلالها استخدام كلا الزيولايتين

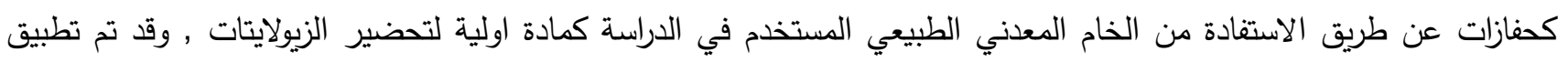
الحفاز في دراسة ستنشر لاحقاً وتظهر قدرته التحفيزية في مجال الاصداح التركيبي الحفازي للمشتق النفطي (النفثا) . الشكر والتقدير

نتقدم بالثكر والعرفان الى عمادة كلية التربية للعلوم الصرفة وقسم الكيمياء في جامعة الموصل لتوفيرهم المواد الكيمياوية والمختبرات لاكمال متطلبات البحث , والى قسم الكيمياء / كلية العلوم في جامعة تكريت لاجرائه بعض القياسات الخاصة بالبحث .

المصادر

1. Auerbach, S.; Carrado, K.A. \& Duta, P.K., (2003), "Handbook of Zeolite Science and Technology", Marcel Dekker, Inc ., p.1-8. 
2. Runer, X., (2007), "Chemistry of Zeolite and Related Porous Materials", John wiley and Sons, Ltd .p.p. 33-34.

3. Hagen, J. (2006),"Industrial Catalysis", Wiley VCH Verlag, Weinheim, Germany, p.p.243-247.

4. Ruren X. ,Wengin P. ,Jihong Y. ,Qisheng H. and Jisheng Ch.,(2007), " Chemistry of Zeolites and Related Porous Materials: Synthesis and Structure ", John Wiley \& Sons (Asia) Pte. Ltd., p.p. 361-364.

5. Mumpton, F.A.,(1999),"Uses of Natural Zeolite in Agriculture and Industry", Edit .Inc., NewYork, 14430,p.1-18.

6. Sels , B.F. and Kustov L.M. , (2016), "Zeolite and Zeolite - Like Materials" , Publications of Elsevier , p.p 265-282 .

7. Yu, S., Yan, J., Lin, W., Zhang, J., and long, J., (2021)," Characterization and Cracking performance of Zirconium- modified Y Zeolite" Catalysis Communications, Vol. (148) No.(106171) .

8. Delgado, A., Robla, J., padilla, I., Andres, S., and Romero, M., (2020), Zero-waste Process for the transformation of a hazardous aluminum waste into a raw material to obtain Zeolites" Journal of Cleaner Production, Vol(255) No.(20).

9. Gadhban, M., Abdulmajed, Y., Ali, F., and Al-Sharify, Z.,(2020), "Preparation of Nano Zeolite and itsApplication in Water Treatment", Materials Science and Engineering Vol.(870) No.(012054).

10. Lee, T., Jeong, H., Jeong, B., Han, J., Gim, M., Kim, D., and Lee, K., (2020),"preparation of HZSM-5 Catalysts with different rations of structure directing agents and their effects on the decomposition of exo-tetrahydrodicyclopentadiene under supercritical conditions and coke formation", Journal Applied Surface Science Vol(511) No.(145398).

11. Araki, S., Okubo, Y., Maekawa, K., Imasaka, S., and Yamamoto,H., (2020)," Preparation of a high-silica chabazite-type zeolite membrane with high $\mathrm{CO} 2$ permeability using tetraethylammonium hydroxide" Journal of Membrane Science, Vol(613) P.P(1-31).

12. Pansu, M. and Gautheyrou, J., (2006), "Handbook of Soil Analysis", Springer-Verlag, p.p.1518,226-233.

13. Adekola, F.A., Olosho, A.I., Baba, A.A. and Adebayo, S.A., (2018), "Dissolution Kinetics Studies of Nigerian Gypsum Ore in Hydrochloric Acid", Journal of Chemical Technology and Metallurgy, Vol.(53), No.(5), p.p.845-855.

14. Loeppert, R.H. and Suarez, D., (1996), "Carbonate and Gypsum ", Publication From USDA ARS / UNL / Faculty, University of Nebraska, Lincoln .

15. Gougazeh, M., (2018), "Removal of Iron and Titanium Contaminants From Jordanian Kaolin's By Using Chemical Leaching", Journal of Taibah University of Science, Vol.(12),No.(3), p.p. 247-254.

16. Nibori, Y ., Kunita, M., Tochiyama, O. and Chida, T., (2000), " Dissolution Rates of Amorphous Silica in Highly Alkaline Solution", Journal of Nuclear Science and Technology, Vol. (37), No. (4), p.p.349-357.

17. Abdul Halim , A.K. , Camer, S.K. and Rahman , Am. , (2010), "Characterization and Cracking Activity of Zeolite Prepared Form Local Kaolin" , Iraqi Journal of Chemical and Petroleum Engineering, Vol. (11), No.(2), p.p. 35-42.

18. Ismail,H .,A.,Ka-Lun, W.,Tau,Ch.L ,BoonS.O and Eng-Poh N. ,(2020), " Metal Chlorides Grafted on SAPO-5 (MClx/SAPO-5)as Reusable and Superior Catalysts for Acylation 2-Methylfuran Under Non-Microwave Instant Heating Condition", Processes, Vol.8,No. 603.

19. American Mineralogist crystal structure database (http://rruff.geo.arizona.edu/AMS/index_min.php).

20. Gabbot , P. , (2008) , "Principles and Applications of Thermal Analysis" , Blackwell Publishing Ltd. , p.p. 88-97 